\title{
Domain wall anisotropic magnetoresistance in planar nanowires
}

L. K. Bogart, and D. Atkinson

Citation: Appl. Phys. Lett. 94, 042511 (2009);

View online: https://doi.org/10.1063/1.3077174

View Table of Contents: http://aip.scitation.org/toc/apl/94/4

Published by the American Institute of Physics

\section{Articles you may be interested in}

Room temperature skyrmion ground state stabilized through interlayer exchange coupling Applied Physics Letters 106, 242404 (2015); 10.1063/1.4922726

Dynamics of antiferromagnetic skyrmion driven by the spin Hall effect Applied Physics Letters 109, 182404 (2016); 10.1063/1.4967006

Magnetic domain-wall creep driven by field and current in $\mathrm{Ta} / \mathrm{CoFeB} / \mathrm{MgO}$ AIP Advances 7, 055918 (2017); 10.1063/1.4974889

Resistivity of Permalloy thin films

Journal of Applied Physics 45, 2780 (2003); 10.1063/1.1663668

Current-induced three-dimensional domain wall propagation in cylindrical NiFe nanowires Journal of Applied Physics 119, 153902 (2016); 10.1063/1.4946753

The design and verification of MuMax3

AIP Advances 4, 107133 (2014); 10.1063/1.4899186

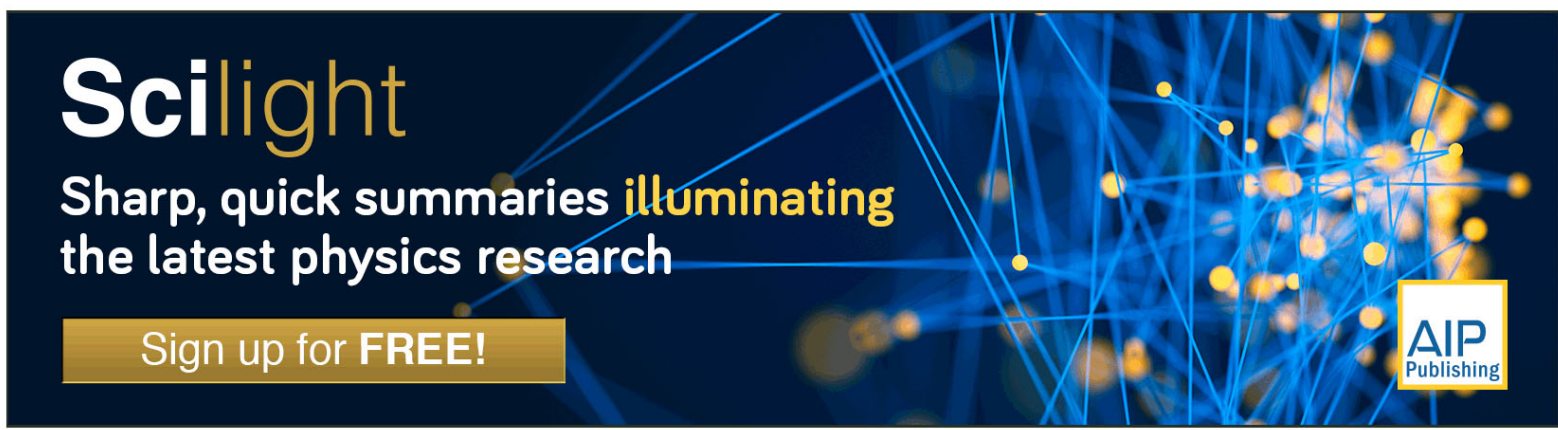




\title{
Domain wall anisotropic magnetoresistance in planar nanowires
}

\author{
L. K. Bogart and D. Atkinson ${ }^{\text {a) }}$ \\ Department of Physics, Durham University, Durham, DH1 3LE, United Kingdom
}

(Received 22 December 2008; accepted 9 January 2009; published online 30 January 2009)

\begin{abstract}
The anisotropic magnetoresistance (AMR) of individual magnetic domain walls in planar nanowires has been systematically investigated as a function of the micromagnetic wall structure, width, and thickness of the nanowire. Experimentally derived thickness dependence of both the thin film resistivity and the anisotropic magnetoresistance were incorporated into the calculations. We found that the AMR value can be used to identify the wall structure and that the wall resistance is sensitive to wire width for widths less than $300 \mathrm{~nm}$. Furthermore, in comparison with the detailed analysis here, a previous simplified domain wall model significantly underestimates domain wall resistance in narrower wires. (C) 2009 American Institute of Physics. [DOI: 10.1063/1.3077174]
\end{abstract}

Magnetic domains walls (DWs) in nanowires are the subject of intensive research both in terms of improving the understanding of the basic physical processes ${ }^{1}$ and also for developing potential applications. ${ }^{2-4}$ In addition to the anisotropic magnetoresistance (AMR) contribution, further spintronic contributions to the electrical resistance of DWs have been proposed. ${ }^{5,6}$ However, in the case of Permalloy nanowires, such contributions to DW resistance may be very small and the magnetoresistance is dominated by the AMR effect. In conventional AMR the resistivity is high when the magnetization and current path are parallel $\left(\rho_{\|}\right)$and is low when the magnetization and current are perpendicular $\left(\rho_{\perp}\right)$. A DW in an axially magnetized nanowire changes the electrical resistance of the nanowire because the magnetization within the wall deviates from the long axis of the wire. AMR offers a convenient technique to identify the presence of a DW since it is based upon electrical measurements, and a DW is detected by the resistance change $\Delta R=R_{\mathrm{DW}}-R_{\mathrm{Sat}}$, where $R_{\mathrm{DW}}$ is the resistance of the nanowire containing a DW and $R_{\text {Sat }}$ is the resistance of the nanowire at saturation. It has recently been reported ${ }^{7,8}$ that both the wall structure (transverse or vortex) and the chirality (sense of magnetization rotation) of a wall pinned at a notch can be identified by subtly different magnetoresistance signatures. However, in the literature there is currently only a simple and somewhat crude model used to interpret the change in resistance $\Delta R$. This is based upon a simplified representation of a DW described by the wall width parameter $\Delta_{\mathrm{DW}}$ (Ref. 9) and overlooks the sophisticated micromagnetic spin structure, which may be important for wires in which different wall structures can exist.

In this letter we present a detailed analysis of the change in resistance due to a DW in Permalloy nanowires as a function of the wire width and thickness; taking both the detailed micromagnetic structure of the wall and the thickness dependence of the electrical resistivity into account. We used a method based on micromagnetic simulations in which the AMR for individual simulation cells is calculated, and mapped the parameter space for nanowires with thicknesses from 3 to $20 \mathrm{~nm}$ and widths from 50 to $600 \mathrm{~nm}$.

Insight into the relationship between DW structure and the magnitude of the resistance change $\Delta R$ in planar nano-

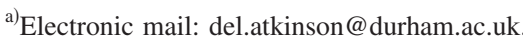

wire structures containing a single wall was obtained using micromagnetic simulations, which were performed using the two-dimensional solver in OOMMF, ${ }^{10}$ and utilized a $5 \mathrm{~nm}$ cell size. Standardized magnetic parameters for Permalloy were used, including saturation magnetization $M_{S}=860$ $\times 10^{3} \mathrm{~A} / \mathrm{m}$ and exchange energy constant $A=13$ $\times 10^{-12} \mathrm{~J} / \mathrm{m}$. Simulations were performed in a quasistatic regime with the damping parameter $\alpha$ set at 0.5 to speed up the convergence time. Using the phase diagram reported in Ref. 11 as a guide, two basic DW structures (transverse and vortex) were investigated. The selected wall type was introduced into a $6000 \mathrm{~nm}$ long rectangular shaped structure with width $w$ and thickness $t$ and was allowed to relax to its equilibrium configuration at zero-applied field. Further simulations were performed at zero field saturation (no wall) for each wire width and thickness. Figure 1(a) presents a schematic of the electrical structure of the nanowire used within this analysis. Each nanowire is represented as a network of conduction channels of resistors in series along the axis of the nanowire; the number of channels is equal to the nanowire width divided by the cell size. Individual resistors correspond to an individual cell within the simulation and the angle $\theta$ between the local magnetization and the current (along the $x$ axis) is used to calculate the resistivity from the relation

$$
\rho(\theta)=\rho_{\perp}+\delta \rho \cos ^{2} \theta .
$$

The total resistivity of the nanowire is obtained from the summation of these conduction channels in parallel and the total magnetoresistance of the nanowire is obtained via $R$ $=\rho L / A$, where $L$ is the length and $A$ is the cross-sectional area of a cell. Thickness dependent values of $\rho_{\|}$and $\rho_{\perp}$ have also been incorporated, however, these are rarely measured directly and have been deduced both from several experimental data sets of $\rho$ and $\delta \rho$ using $\delta \rho=\rho_{\|}-\rho_{\perp}$ and $\rho$ $\left.=1 / 3\left(\rho_{\|}+2 \rho_{\perp}\right)\right)^{9,12}$ Figure $1(\mathrm{~b})$ presents resistivity data as a function of film thickness below $50 \mathrm{~nm}$ for $\mathrm{Ni}_{82} \mathrm{Fe}_{18}$ films measured by several workers ${ }^{13-16}$ and data measured here for nominally $\mathrm{Ni}_{81} \mathrm{Fe}_{19}$ films. Each set of data shows that as film thickness decreases the resistivity increases, as is expected since the resistivity increases noticeably when the mean free path, $l_{o}$, of the conduction electrons is comparable to film thickness, $t$, due to diffuse scatter at the surfaces. ${ }^{17}$ The data are consistent across these studies, although the largest dif- 


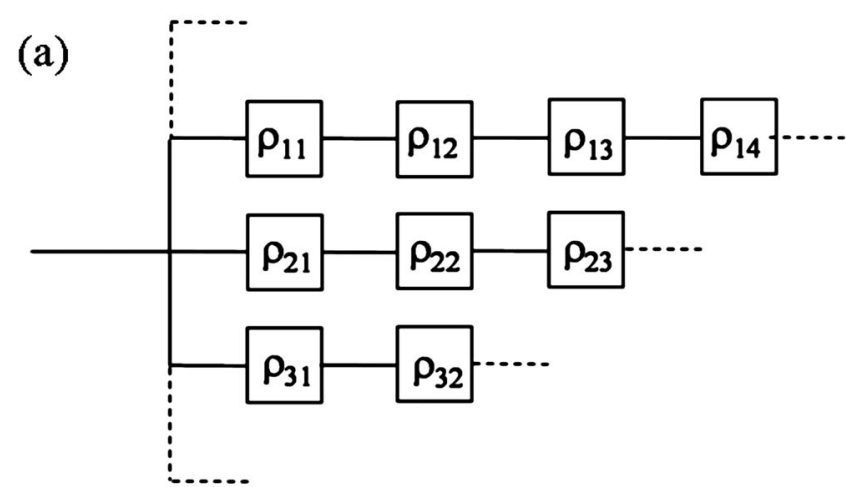

(a)
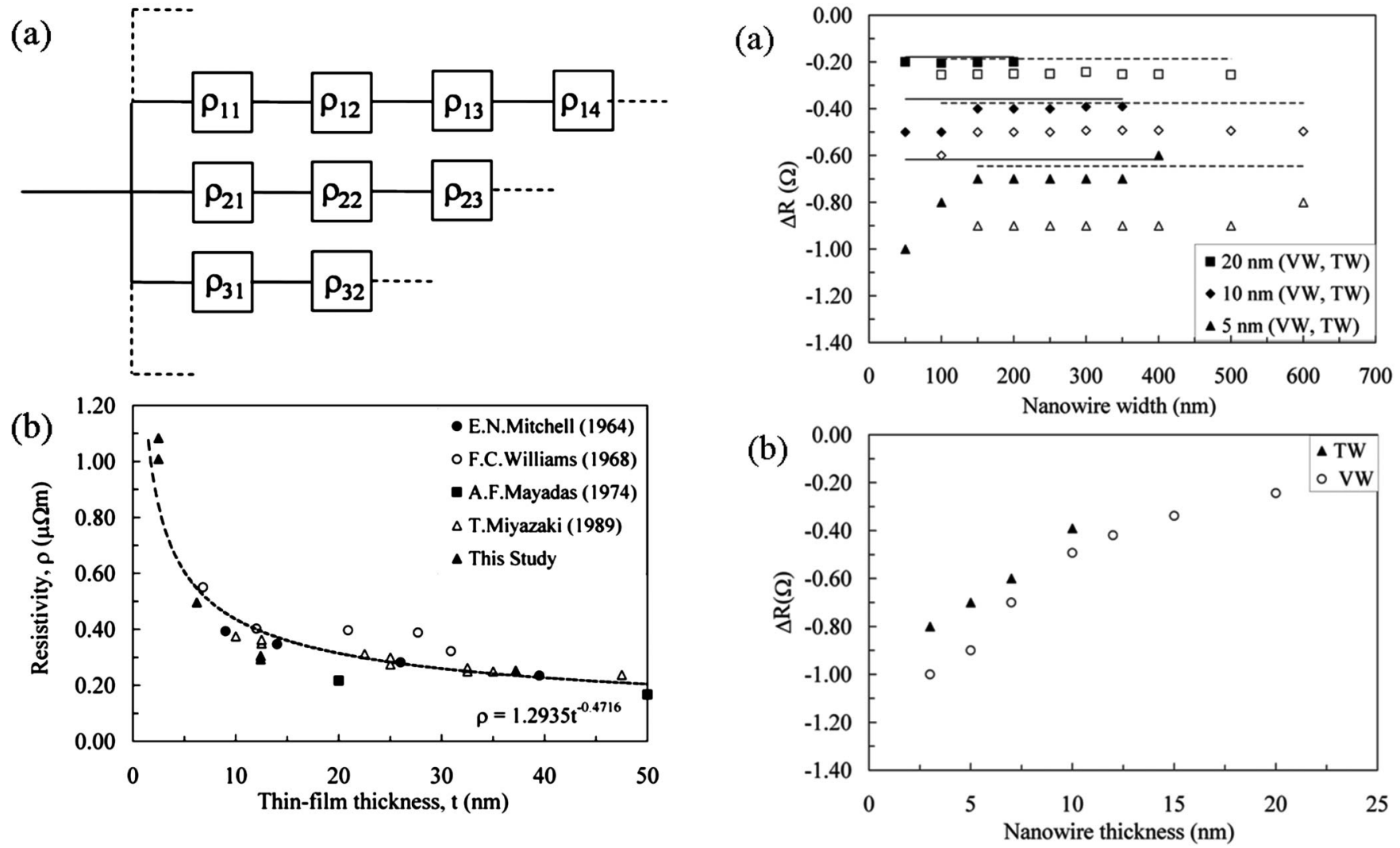

FIG. 1. (a) Schematic of the electrical network representing a nanowire used to calculate the resistance. Each resistor represents a cell from the micromagnetic simulation. (b) Comparison of the thickness dependent resistivity of five data sets for Permalloy. The line is the best fit to all of the data.

ferences occurring between 20 and $30 \mathrm{~nm}$ for the Williams and Mitchell ${ }^{14}$ data are attributed to the differences in the deposition rates used during film preparation. A line of best fit to all the plotted data yields a thickness dependence of the resistivity given by $\rho=1.2935 t^{-0.4716}$, where $t$ is measured in nanometers and $\rho$ is given in $\mu \Omega \mathrm{m}$. Literature data for $\delta \rho$ are more limited, ${ }^{13-15}$ in which for $t<50 \mathrm{~nm}$ a weak logarithmic dependence of $\delta \rho$ upon thickness is observed. The thickness trend of the data is similar between the studies but there is some variation in magnitude, which again may be attributed to the preparation method. The best fit to the experimental data is given by $\delta \rho / \rho=0.7057 \ln (t)-0.3356$. In contrast with the thickness dependence, the wire width dependence of the resistivity can be ignored since the length at which such effects emerge is below $45-50 \mathrm{~nm} .^{18}$

Figure 2(a) shows the AMR DW resistance calculated from micromagnetic simulations for both transverse and vortex wall structures as a function of wire width for different thicknesses. The majority of DWs shown are favorable (lowest energy) structures. However we also included unfavorable (higher energy) structures up to only $20 \%$ higher energy to reflect the possible metastable wall structures that may exist in real nanowire samples. Values of the DW resistance deduced from the simple model are shown for comparison and are indicated by the solid (dashed) line for transverse (vortex) walls. The simple model utilizes $\Delta R=R_{\mathrm{DW}}-R_{\mathrm{Sat}}$ $=-\delta \rho 2 \Delta_{\mathrm{DW}} / w t$ (Ref. 9), where the wall width parameter $\Delta_{\mathrm{DW}}$ is $\Delta_{\mathrm{TW}}=w / \pi$ for transverse walls and $\Delta_{\mathrm{VW}}=3 w / 4$ for vortex walls. ${ }^{11}$ There are significant differences between the two approaches with the simple model considerably underestimating the AMR value, which is particularly noticeable

FIG. 2. (a) Calculated DW resistance as a function of wire width for 5, 10 , and $20 \mathrm{~nm}$ thick nanowires and comparison with values obtained from the 1D wall model. Solid symbols (line) correspond to transverse walls and open (dashed) symbols (line) to vortex walls. (b) Thickness dependence of the DW resistance for a $300 \mathrm{~nm}$ wide wire from both transverse and vortex walls.

for vortex DWs in 5 and $10 \mathrm{~nm}$ thick nanowires, where the difference between the simple model and our detailed approach is up to $40 \%$. For thicker films the values predicted by the two models differ less, although the simple model still underestimates the magnitude of $\Delta R$, especially for vortex walls. It can be seen in Fig. 2(a) that the simple model predicts that for a given thickness, $\Delta R$ is independent of wire width, which is a direct result of the one-dimensional (1D) Bloch wall width definitions. Our modeling, however, shows that, for narrower widths, $\Delta R$ has a marked dependence on wire width. These differences are attributed to the wall width parameter, which simplifies the DW and neglects the sophisticated micromagnetic spin structure, in particular, in narrow nanowires where large numbers of high angle (low resistance) spins are contained within the wall. The present analysis shows that the dependence of the wall resistance on wall structure is significantly larger than previously predicted. Figure 2(b) shows the AMR wall resistance for a $300 \mathrm{~nm}$ wide wire as a function of film thickness. The energetically favored wall structure depends sensitively upon wire thickness, with vortex walls favored in thicker nanowires, transverse walls in the thinnest wires, and both wall structures possible at intermediate thicknesses. From Fig. 2(b) it is clear that the resistance of a transverse wall is smaller than that of a vortex wall, and this is attributed to vortex walls being larger and hence containing more off-axis spins.

In summary, it is found that the variation in wall resistance is more complex than that derived from an earlier simple model. For thin, narrow nanowires the simple model 
significantly underestimates $\Delta R$ by more than $40 \%$ compared to the detailed analysis presented here. Finally, for a given nanowire the difference between the DW resistance of vortex and transverse walls is found to be larger than that predicted by the simpler model.

${ }^{1}$ C. H. Marrows, Adv. Phys. (Berlin) 54, 585 (2005).

${ }^{2}$ S. S. P. Parkin, M. Hayashi, and L. Thomas, Science 320, 190 (2008).

${ }^{3}$ M. Diegel, R. Mattheis, and E. Halder, IEEE Trans. Magn. 40, 2655 (2004).

${ }^{4}$ D. Atkinson, D. S. Eastwood, and L. K. Bogart, Appl. Phys. Lett. 92, 022510 (2008).

${ }^{5}$ P. M. Levy and S. Zhang, Phys. Rev. Lett. 79, 5110 (1997).

${ }^{6}$ G. Tatara and H. Fukuyama, Phys. Rev. Lett. 78, 3773 (1997).

${ }^{7}$ M. Hayashi, L. Thomas, R. Moriya, C. Rettner, and S. S. P. Parkin, Nat. Phys. 3, 21 (2007).
${ }^{8}$ M. Hayashi, L. Thomas, C. Rettner, R. Moriya, X. Jiang, and S. S. P. Parkin, Phys. Rev. Lett. 97, 207205 (2006).

${ }^{9}$ L. Thomas and S. S. P. Parkin, in Handbook of Magnetism and Advanced Magnetic Materials, edited by H. Kronmüller and S. S. P. Parkin (Wiley, Chichester, 2007), Vol. 2, pp. 942-982.

${ }^{10}$ http://math.nist.gov/oommf/.

${ }^{11}$ Y. Nakatani, A. Thiaville, and J. Miltat, J. Magn. Magn. Mater. 290, 750 (2005).

${ }^{12}$ T. R. McGuire and R. I. Potter, IEEE Trans. Magn. 11, 1018 (1975).

${ }^{13}$ E. N. Mitchell, H. B. Haukaas, H. D. Bale, and J. B. Streeper, J. Appl. Phys. 35, 2604 (1964).

${ }^{14}$ F. C. Williams, Jr. and E. N. Mitchell, Jpn. J. Appl. Phys. 7, 739 (1968)

${ }^{15}$ F. Mayadas, J. F. Janak, and A. Gangulee, J. Appl. Phys. 45, 2780 (1974).

${ }^{16}$ T. Miyazaki, T. Ajima, and F. Sato, J. Magn. Magn. Mater. 81, 86 (1989).

${ }^{17}$ K. Fuchs, Proc. Cambridge Philos. Soc. 34, 100 (1938).

${ }^{18}$ C. Durkan and M. E. Welland, Phys. Rev. B 61, 14215 (2000). 